\title{
The long QT syndrome family of cardiac ion channelopathies: A HuGE review*
}

Stephen M. Modell, MD, MS ${ }^{1}$, and Michael H. Lehmann, $M D^{2}$

\begin{abstract}
Long QT syndrome (LQTS) refers to a group of "channelopathies"-disorders that affect cardiac ion channels. The "family" concept of syndromes has been applied to the multiple LQTS genotypes, LQT1-8, which exhibit converging mechanisms leading to QT prolongation and slowed ventricular repolarization. The $470+$ allelic mutations induce loss-of-function in the passage of mainly $\mathrm{K}^{+}$ions, and gain-of-function in the passage of $\mathrm{Na}^{+}$ions through their respective ion channels. Resultant early after depolarizations can lead to a polymorphic form of ventricular tachycardia known as torsade de pointes, resulting in syncope, sudden cardiac death, or near-death (i.e., cardiac arrest aborted either spontaneously or with external defibrillation). LQTS may be either congenital or acquired. The genetic epidemiology of both forms can vary with subpopulation depending on the allele, but as a whole, LQTS appears in every corner of the globe. Many polymorphisms, such as HERG P448R and A915V in Asians, and SCN5A $\mathrm{S} 1102 \mathrm{Y}$ in African Americans, show racial-ethnic specificity. At least nine genetic polymorphisms may enhance susceptibility to drug-induced arrhythmia (an "acquired" form of LQTS). Studies have generally demonstrated greater QT prolongation and more severe outcomes among adult females. Gene-gene interactions, e.g., between SCN5A Q1077del mutations and the SCN5A H558B polymorphism, have been shown to seriously reduce ion channel current. While phenotypic ascertainment remains a mainstay in the clinical setting, SSCP and DHPLC-aided DNA sequencing are a standard part of mutational investigation, and direct sequencing on a limited basis is now commercially available for patient diagnosis. Genet Med 2006:8(3):143-155.
\end{abstract}

Key Words: Long QT syndrome, ion channelopathies, torsade de pointes, epidemiology, review

\section{GENES AND CORRESPONDING ELECTROPHYSIOLOGY}

The congenital (also called "idiopathic") form of long QT syndrome (LQTS) is mainly caused by mutations in genes that code for protein subunits of cardiac ion channels. As of May, 2005, eight major genotypes, LQT1-8, 471 different mutations, and 124 polymorphisms were described in the European Society of Cardiology Working Group on Arrhythmias (WGA) LQTS gene database http://pc4.fsm.it:81/cardmoc/main.htm. Among the various LQTS genotypes, the most common feature predisposing to arrhythmia is prolongation of the ventricular action potential duration during cardiac repolarization, measured as the QT interval on the electrocardiogram (Fig. 1), ${ }^{1}$ which can lead to early after-depolarizations and life-threatening torsade de pointes (TdP)(Fig. 2). ${ }^{2}$ This converging mechanism has led some to ascribe the "family" concept to the various LQTS genotypes, ${ }^{3}$ though considerable heterogeneity ex-

From the ${ }^{1}$ Department of Health Management and Policy, University of Michigan School of Public Health; ${ }^{2}$ Department of Internal Medicine, Division of Cardiovascular Medicine, University of Michigan Medical System.

Stephen M. Modell, MD, MS, University of Michigan, School of Public Health, M-4157, OCBPH, SPH-II, 109 S. Observatory, Ann Arbor, MI 48109-2029. E-mail:mod@umich.edu

Submitted for publication July 27, 2005.

Accepted for publication December 16, 2005.

${ }^{*} A$ more extensive version of this article with a more complete list of references is available at www.geneticsinmedicine.org.

DOI: 10.1097/01.gim.0000204468.85308.86 ists. Table 1 depicts the range of genes composing the idiopathic "long QT syndromes" and their corresponding electrophysiology. ${ }^{4-6}$

\section{Cellular electrophysiology}

The ventricular action potential proceeds through five phases (cardiac action potential; Fig. 3). ${ }^{3}$ The initial upstroke (phase 0 -depolarization) occurs through the opening and closing of $\mathrm{Na}^{+}$channels. The repolarization process begins with the rapid transient outflow of $\mathrm{K}^{+}$ions (phase $1-\mathrm{I}_{\text {to }}$ current). This is followed by the flow of outward current through two delayed rectifier $\mathrm{K}^{+}$channels $\left(\mathrm{I}_{\mathrm{Ks}}, \mathrm{I}_{\mathrm{Kr}}\right)$ and of inward current through $\mathrm{Ca}^{2+}$ channels, constituting phase 2 or the plateau phase of repolarization. Increasing conductance of the rapid delayed rectifier $\left(\mathrm{I}_{\mathrm{Kr}}\right)$ and inward rectifier $\left(\mathrm{I}_{\mathrm{K} 1}\right)$ currents completes repolarization (phase 3 ). Phase 4 represents a return of the action potential to baseline.

\section{Specific LQTS genotypes}

The LQT1 gene (also known as KCNQ1 and KvLQT1) spans $400 \mathrm{~kb}$ and encodes voltage-gated potassium channel alpha subunits. A tetramer of $4 \mathrm{KCNQ} 1$ alpha subunits co-assembles with the minK gene product (beta regulatory subunit) to form the $\mathrm{I}_{\mathrm{Ks}}$ slowly deactivating delayed rectifier potassium channel. ${ }^{4}$ At least $179 \mathrm{KCNQ} 1$, mostly missense, mutations have been reported in the WGA database. For potassium channel muta- 


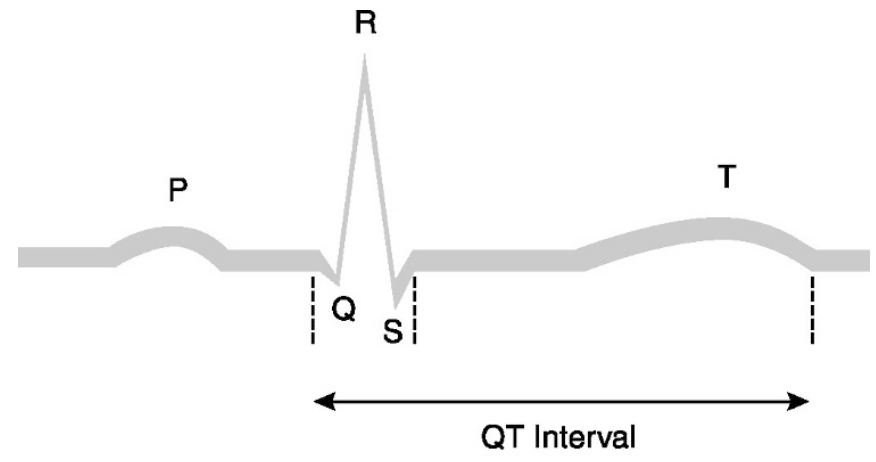

Rate-correction formula (Bazett's): QTc $(\mathrm{msec})=\frac{\mathrm{QT}(\mathrm{msec})}{\sqrt{\mathrm{RR}(\mathrm{sec})}}$

Fig. 1. Measurement of the QT interval from the electrocardiogram (ECG). Diagram depicts the normal ECG with P-wave representing atrial activation, QRS complex representing ventricular activation and the start of ventricular contraction, and T-wave representing ventricular repolarization. The QT interval is defined as the distance from the beginning of the Q-wave to the end of the T-wave. Owing to heart rate sensitivity of the QT interval, this parameter is corrected (normalized) to heart rate (as reflected by the beat-to-beat "RR" interval), yielding the "QTc." QT and QTc can both be expressed in msec or sec. Adapted from Liu BA, Juurlink DN. N Engl J Med 2004, ${ }^{1}$ with premission of the Massachusetts Medical Society. (C)2004. All rights reserved.

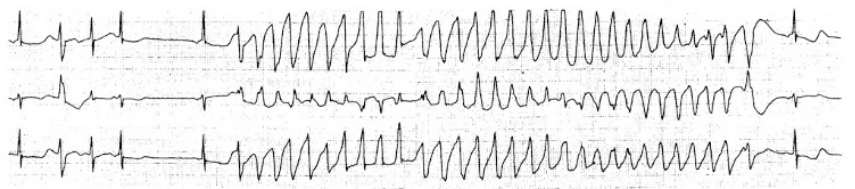

Fig. 2. Arrhythmia onset in long QT syndrome. Three simultaneous (and distinct) ECG channel recordings in a patient with QT prolongation and runs of continuously varying polymorphic ventricular tachycardia (torsade de pointes [TdP]). TdP may resolve spontaneously or progress to ventricular fibrillation and cardiac arrest. Reproduced from Chiang C, Roden DM. J Am Coll Cardiol 2000, ${ }^{2}$ with permission of the American College of Cardiology Foundation. (C2000. All rights reserved.

tions in general, mixed alpha subunit tetramers (wild-type plus mutated units) exhibit abnormal protein function, producing a dominant-negative effect on ion channel current. The net effect of LQT1 mutations is a decreased outward $\mathrm{K}^{+}$current during the plateau phase of the cardiac action potential, i.e., a loss-of-function of the ion channel. The channel remains open longer, ventricular repolarization is delayed, and the QT interval is lengthened.

The gene for LQT2 (also known as the KCNH2 and human ether-a-go-go-related or HERG gene) spans $55 \mathrm{~kb}$ and also encodes potassium channel alpha subunits. Tetramers of these subunits form the $\mathrm{I}_{\mathrm{Kr}}$ rapidly activating, rapidly deactivating delayed rectifier potassium channel, which associates with the minK-related peptide 1 (MiRP1) gene product. ${ }^{4}$ At least 198 distinct HERG mutations have been identified. The majority of HERG pore region defects are missense mutations, while nonpore defects demonstrate a variety of missense, nonsense, and frameshift mutations. Mutations can result in either structural ion channel defects having dominant-negative effects or intra- cellular "trafficking" abnormalities causing a reduction in the number of functional ion channels, both leading to loss-offunction. In terms of electrophysiology, HERG mutations cause potassium ion channels to deactivate (close) much faster, blunting the normal rise in current $\left(\mathrm{I}_{\mathrm{Kr}}\right)$ that results from rapid recovery from channel inactivation/slow deactivation. The $\mathrm{I}_{\mathrm{Kr}}$ current during the plateau phase is reduced and ventricular repolarization delayed, leading to QT interval prolongation.

The LQT3 gene, SCN5A, spans $80 \mathrm{~kb}$. At least 56 LQTS SCN5A mutations have been identified. The SCN5A alpha subunit, comprised of four sequential domains that fold endto-end into a torus-like shape, can form a fully functional channel; beta subunits have a modifying influence. LQT3 mutations lead to the reopening of sodium channels (i.e., gain-offunction), thereby enhancing the inward plateau current and prolonging repolarization.

The gene responsible for LQT4, $220 \mathrm{~kb}$ in length, encodes the ankyrin-B (ANKB or ANK2) "adaptor" protein. The 5 reported AnkB mutations interfere with anchoring of $\mathrm{Na}, \mathrm{K}$ ATPase and the $\mathrm{Na} / \mathrm{Ca}$ exchanger, resulting in $\mathrm{Na}^{+}$build-up and a compensatory increase in intracellular $\mathrm{Ca}^{2+}$ stores. Though fatal arrhythmias can occur, QTc (the heart-rate corrected QT interval ${ }^{1}$ ) is inconsistently prolonged.

The relatively small minK gene, mutations in which cause LQT5, is $40 \mathrm{~kb}$ in length. The encoded KCNE1 protein contains a single transmembrane spanning domain with small intra- and extracellular components. The product of the $\operatorname{minK}$ gene forms the beta subunit of the LQT1 assembly regulating the $\mathrm{I}_{\mathrm{Ks}}$ potassium channel current.

The LQT6 gene encoding MiRP1, or minK-related protein 1 , is located $70 \mathrm{~kb}$ from minK on the same chromosome. The two genes bear many similarities, suggesting a common evolutionary origin, possibly from a duplication event. The MiRP1 gene product KCNE2 coassembles as the beta subunit with HERG alpha subunits to regulate $\mathrm{I}_{\mathrm{Kr}}$ potassium currents.

The LQT7 genotype has been mapped to the inward rectifying potassium channel gene KCNJ2 on chromosome 17. KCNJ2 encodes the potassium channel protein Kir2.1. Kir2.1 plays an important role in the generation of inward repolarizing $\left(\mathrm{I}_{\mathrm{K} 1}\right)$ currents during the terminal stages (phase 3 ) of the cardiac action potential. Patients generally exhibit relatively modest QTc prolongation, rarely degenerating into TdP and its sequellae, though more malignant, life-threatening mutations (R67W and C101R) have been observed.

The most recent addition, LQT8, has been mapped to the calcium channel gene CACNA1C on chromosome 12. CACNA1C mutations cause nearly complete loss of voltage-dependent L-type $\mathrm{Ca}_{\mathrm{v}} 1.2$ channel inactivation, affecting the inward (depolarizing) $\mathrm{Ca}^{2+}$ current during the plateau phase (phase 2). Marked QT interval prolongation (as high as 730 milliseconds) and fatal arrhythmias (often in the first three years) are characteristic. All individuals tested so far have had de novo mutations.

LQT1, 3, and 4 genes have also been implicated as possible quantitative trait loci in non-LQTS study participants. Heritability estimates of genetic factors explaining QT interval variation in the freestanding population range between 25 and $60 \%$. 


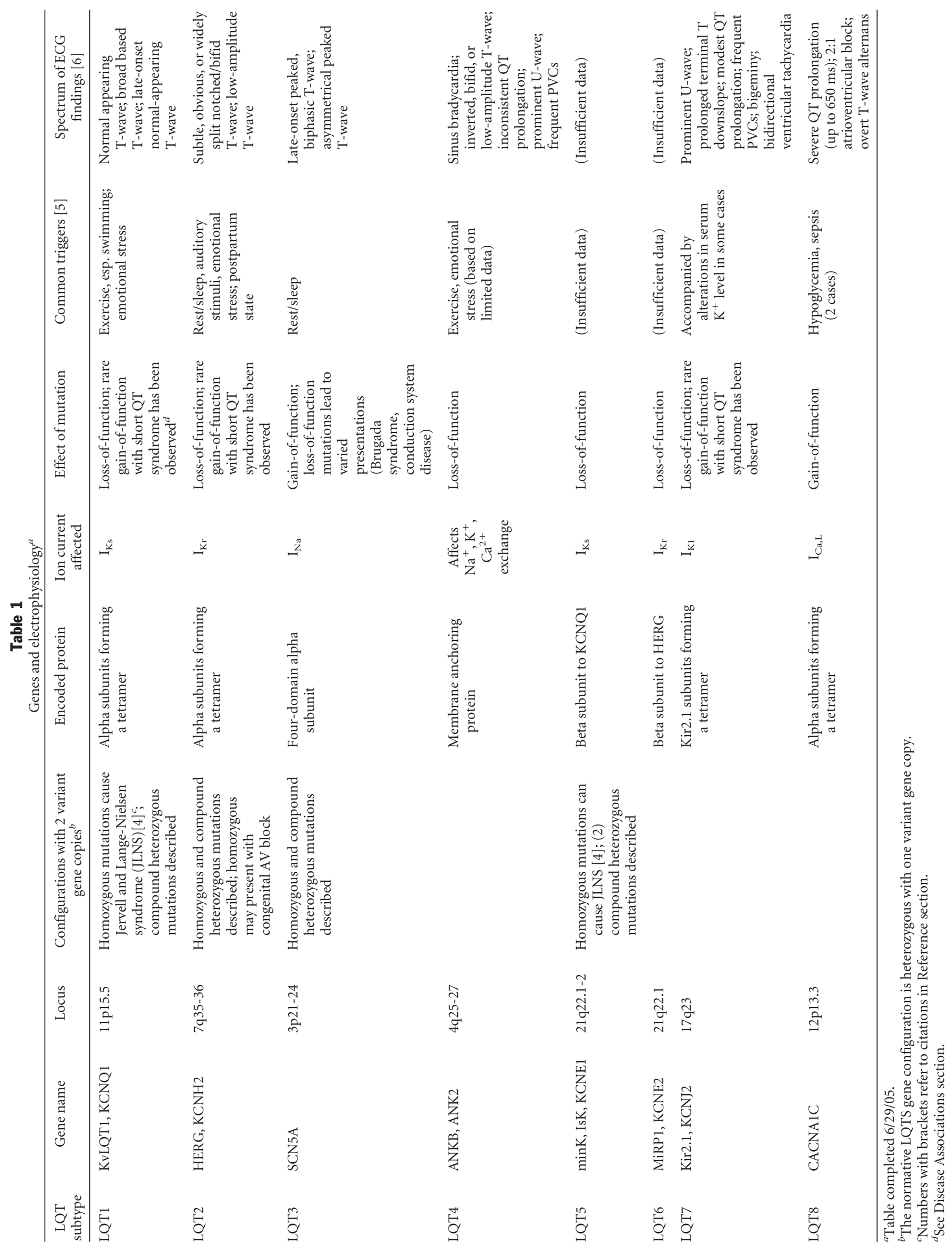




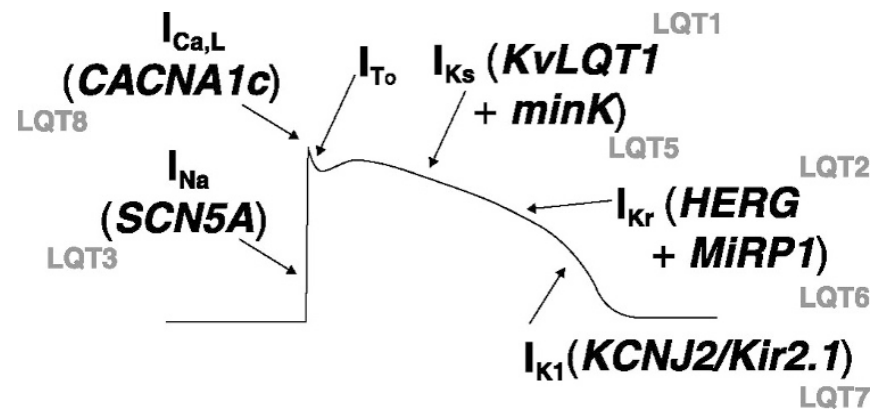

Fig. 3. Ionic currents/LQT gene products and the ventricular action potential. Vertical axis represents voltage; horizontal axis represents time. The initial upstroke (phase 0), rapid depolarization, occurs through the rapid inflow of sodium $\left(\mathrm{I}_{\mathrm{Na}}\right)$. The peak, rapid repolarization (phase 1 ), is mediated by a transient outward potassium current, $\mathrm{I}_{\text {to }}$. The plateau (phase 2) reflects a balance between inward sodium and L-type calcium currents $\left(\mathrm{I}_{\mathrm{Ca}, \mathrm{L}}\right)$, and outward potassium $\left(\mathrm{I}_{\mathrm{Ks}}, \mathrm{I}_{\mathrm{Kr}}\right)$ and chloride currents (not shown). Increasing conductance of rapid potassium current $\left(\mathrm{I}_{\mathrm{Kr}}\right)$ completes repolarization (phase 3 ) together with contributions from the slow potassium current $\left(\mathrm{I}_{\mathrm{Ks}}\right)$ and inward rectifying current $\left(I_{K 1}\right)$. The cell then returns to its baseline resting potential (phase 4) until the next upstroke. The LQT gene products depicted in parentheses encode most of the ion channe proteins mediating these currents. Modified from Roden DM, Spooner PM. J Cardiovasc Electrophysiol 1999, ${ }^{3}$ with permission of Blackwell.

\section{GENE VARIANTS}

\section{Patterns in LQTS mutations}

We conducted a MedSearch of the LQTS literature from major regions of each continent for the period 1975-2004 and reviewed the mutation and polymorphism-related citations on the WGA and international Human Genome Organization (HUGO) web sites (tables and references can be viewed at www.geneticsinmedicine.org). We excluded mutations not referenced in the last nine years, having incomplete tabular data, or representing nonfunctional intronic variants. The expanse of human subject-related articles demonstrates the presence of LQTS in virtually every corner of the globe.

Mutational site may affect severity of the LQTS phenotype. International Long QT Syndrome Registry patients and kin with LQT2 pore mutations appear to be at a higher risk for cardiac events than individuals with non-pore mutations. Though some studies have failed to demonstrate a consistent difference in phenotypic severity between pore versus nonpore mutational sites in LQT1, a five-center, 95 patient study in Japan indicated QTc may be prolonged to a greater extent in pore versus pre-pore mutations. ${ }^{7}$ A mutational screening survey in the U.S. of 541 unrelated, consecutive LQTS patients performed by Tester et al. failed to corroborate the above LQT1 and 2 relationships. ${ }^{8}$ Additional research is needed to resolve the disparate findings.

Cases and families bearing the same mutation may be separated by considerable distance, e.g., the HERG A614V missense mutation detected in Japanese families and multiple unrelated families of European descent and the HERG S818L missense mutation detected in unrelated families from Belgium and Ireland. In many instances these recurrent genetic events are considered sporadic. However, alternative explanations exist. Tranebjaerg et al., in identifying a JLNS R518X mutation on two different haplotypes in Norwegian families of Swedish and
Scottish ancestry, considered it reflective of either recurrent mutational events or founder effects. ${ }^{9}$ Four alleles studied in the Finnish population-KCNQ1 G589D and IVS7-2A->G (KCNQ1-FinA and B, respectively), and HERG L552S and R176W (HERG-FinA and B, respectively)_represent founder mutations enriched by geographic isolation. ${ }^{10,11}$

Authors have also reported detecting mutational hotspots, coding regions especially prone to harboring various mutations: KCNQ1 A246V (initially labeled A212V; reported in USA and South Africa), A341E and A341V (reported in USA and France), A344A (France), G589D (Scandinavia); HERG A561V (Italy), SCN5A E1784K (USA), 1795insD (the Netherlands); and CACNA1C G406R (USA). Accidental deamination of $\mathrm{CpG}$ dinucleotides at the affected sites may be involved. Some mutations may represent the movement of families with common ancestry.

Regions that are conserved throughout species and that code for an amino acid whose alteration has a severe functional consequence tend to display lower mutational frequencies. ${ }^{10}$ Conversely, allelic changes which escape strongly dominant-negative effects to yield partially functional ion channels, such as the Finnish KCNQ1 G589D mutation, ${ }^{10}$ the HERG A490T missense mutation reported in Japan, ${ }^{12}$ and the KCNE1 V109I mutation described by Schulze-Bahr in Germany, ${ }^{13}$ can persist in families and in populations despite triggering arrhythmias under relatively limited circumstances.

\section{Genotype frequencies}

Table 2 (Refs. 8, 14, 15) lists frequencies for the five most prevalent LQTS genotypes based on pooled patient data and the WGA database. Population-based figures derive from clinics in North America (e.g., Mayo Clinic) and Europe, with patient groups not limited to a particular gender or racialethnic group. Proportions of patients bearing abnormal alleles, from studies in the U.S. (272 LQTS positive patients) and the Netherlands plus Belgium (31 LQTS positive), are: $88.2 \%$ (95\% C.I.: 85.9; 90.4\%) heterozygous; 8.6\% (4.5; 12.7\%) compound heterozygous; and $3.2 \%(0 ; 9.6 \%)$ homozygous. ${ }^{8,16}$ The LQTS literature has only scattered references to homozygosity rates for specific mutations and polymorphisms, though heterozygous rates for LQTS polymorphisms are widely reported.

\section{Patterns in LQTS gene polymorphisms}

Rates for polymorphisms may appear either as population allele (allelic total denominator) or heterozygote (combinatorial denominator) frequencies. Laitinen reports a heterozygous frequency of 0.25 and an allele frequency of 0.16 for the HERG K897T polymorphism in the Finnish population. ${ }^{17}$ The average allele frequency for the 20 LQT1-3 polymorphisms summarized by Iwasa in Japan was 0.14 (range: 0.005 to 0.46 ). ${ }^{18}$

Alterations in LQTS protein products associated with genetic polymorphisms generally exhibit weak suppressive effects in the case of outward potassium currents. For example, the KCNQ1 G643S amino acid polymorphism found in the Japanese population leads to a $30 \%$ reduction in $\mathrm{I}_{\mathrm{Ks}}$ current without much alteration in kinetic properties. ${ }^{19}$ Reductions in 


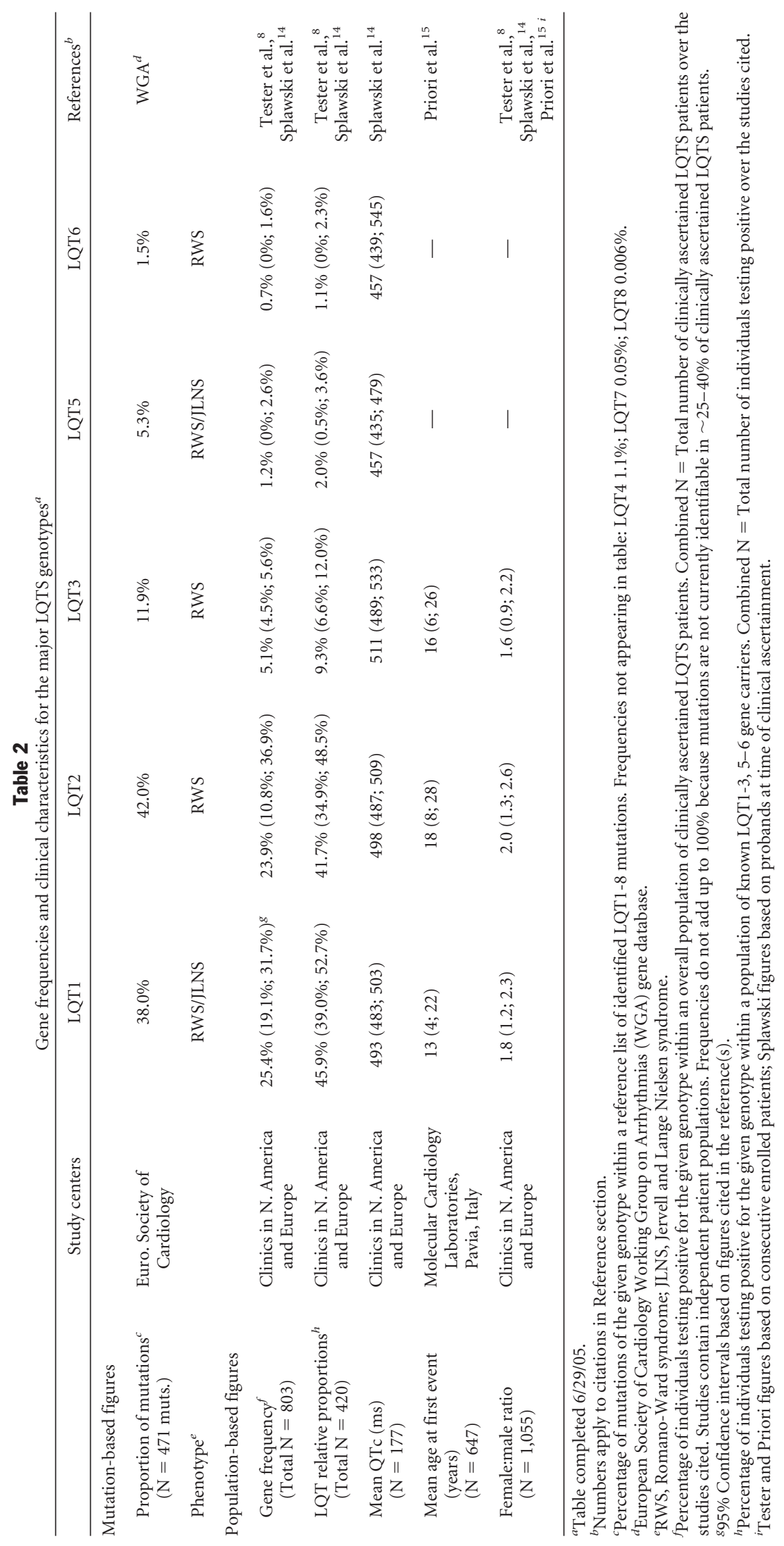


$\mathrm{I}_{\mathrm{Kr}}$ current with the HERG K897T polymorphism are on the order of $10-30 \%$, leading to only subtle increments in action potential duration. ${ }^{20}$ Oftentimes additional factors are required for manifestation of symptoms.

Like many LQTS mutations, numerous polymorphisms have been identified in vastly separate ancestral and geographic populations. Allele frequencies in some instances may be statistically similar and bear further explanation, as in the SCN5A D1819D polymorphism shared by the Japanese and Han Chinese ( 0.46 vs. $0.41, P>0.5){ }^{18,21}$ Many of the polymorphisms reiterated throughout studies exist broadly in their host populations. The HERG K897T and KCNE1 G38S polymorphisms exist in heterozygous form in more than $7 \%$ of each of the four major U.S. racial-ethnic groups (Caucasian, African-American, Latino, and Asian-American), and have been studied internationally. ${ }^{17,22}$

Particular groups may also display higher frequencies of certain allelic variants. For example, the KCNQ1 variant $\mathrm{P} 448 \mathrm{R}$ has been identified in 22 to $28 \%$ of subjects in Japan, but in only $3 \%$ of African American subjects in the U.S., and not in the other two racial-ethnic groups. ${ }^{22,23}$ Splawski et al. noted the SCN5A S1102Y (alternatively known as S1103Y ${ }^{24}$ ) polymorphism, which appears to be associated with drug-related QT prolongation, in $13.2 \%$ of healthy African American cardiovascular study controls. It was also detected in 19.2\% of West African and Caribbean controls. ${ }^{25}$

Systematic racial-ethnic studies have been conducted. Ackerman et al., in looking at genetic repository samples from 744 healthy individuals representing the four major U.S. racial-ethnic groups, discovered that $86 \%$ of the cardiac potassium channel genetic variants were ethnicity specific. For example, the KCNQ1 G643S polymorphism was identified at higher rates in African-American and Asian participants (heterozygous frequencies of $5.9 \%$ and $6.0 \%$, respectively) than in Caucasians and Latinos (0\% and $1.1 \%)$. The heterozygous frequency for HERG P448R was appreciably higher in Asian-Americans (16.4\%) than the other groups (next highest: African-Americans-0.3\%). HERG A915V was identified only in Asian participants (4.5\%). Six of the potassium channel allelic variants that were identified with higher frequency in specific racial-ethnic categories-KCNE1 V109I and KCNE2 Q9E in African-Americans; HERG N33T, R176W, P347S, and P917L in Caucasians-are reported in the literature as potentially pathogenic. ${ }^{22}$ In follow-up studies of sodium channel variants in 829 healthy individuals, 3 of the variants identified that show similar specificities-SCN5A S1102Y in African-Americans; R1193Q in the Japanese; and V1951L in Latinos-are also reported as potentially pathogenic. ${ }^{24}$ Other than the U.S. study by Splawski et al., ${ }^{25}$ comparable data in the general LQTS literature on individuals of original African descent is lacking.

\section{DISEASE}

\section{Trends in disease severity}

The public health importance of LQTS is highlighted by the fact that it can result in sudden death, causing as many as 3,000 unexpected deaths in children and young adults annually in the U.S. ${ }^{4}$ In the absence of treatment, 6 to $13 \%$ of affected individuals succumb to cardiac arrest or sudden cardiac death (SCD) before the age of 40 years. ${ }^{15}$ Demographic characteristics among patient groups, such as sex, age at first event, and mean age first seen in clinic for the event, tend to vary by genotype (Table 2)..$^{14,15,26}$ The frequency of cardiac events (syncope, aborted cardiac arrest and sudden death) tends to be higher in individuals with LQT2 mutations than in those with LQT1 or LQT3 mutations. However, a higher percentage of lethal events by age 40 is associated with the LQT3 genotype. ${ }^{26}$

\section{Inheritance patterns and complexity of disease presentation}

Inherited LQTS manifests in two different forms. The more common familial form, referred to as the Romano-Ward syndrome (RWS), displays the cardiac electrophysiologic abnormalities of LQTS and normal hearing. It is inherited in autosomal dominant fashion. The exact prevalence is unknown, but is frequently approximated at 1 gene carrier in 7,000 persons in the general U.S. population. ${ }^{4}$ A critical single gene mutation in any one of the various LQTS genotypes (particularly LQT1-3) can lead to RWS.

The second familial form, Jervell and Lange-Nielsen syndrome (JLNS), is associated with homozygous KCNQ1 and KCNE1 mutations. JLNS is characterized by marked QT prolongation with a high incidence of sudden death and bilateral sensori-neural deafness. The latter is a consequence of developmental abnormalities in the endolymph-producing stria vascularis of the cochlea, resulting in potassium level disturbances in the inner ear fluid. Prognosis is generally worse than with RWS due to the presence of two copies of the same allele. ${ }^{4}$ The cardiac component is inherited in autosomal dominant fashion, but low penetrance ( $25 \%$ in one three-generation study) in parents of probands may mimic autosomal recessive transmission. ${ }^{27}$ The characteristic of deafness is inherited as an autosomal recessive trait. JLNS is fairly rare in the general population; one study estimated the prevalence in children aged 4 to 19 years in England, Wales and Ireland to be 1.6 to 6 per million. ${ }^{9}$ Prevalance of JLNS in the congenitally deaf ranges from $0.57 \%$ to $6.5 \%$ in the international LQTS literature.

A number of mutations leading to JLNS-572-576del and R518X in KCNQ1, and D76N in KCNE1-have received widespread attention. Tyson et al. note that frameshift/truncating mutations affecting the C-terminal domain of KCNQ1 represent a large number of JLNS mutations, in contrast to the more prevalent missense mutations of RWS, spread throughout a variety of domains. ${ }^{28}$ Double mutants (homozygotes) of LQT2 and 3, yielding ion channel knock-outs, exhibit a more severe phenotype than their single gene counterparts, and can result in intrauterine and neonatal complications.

The effect of a given mutation can be potentiated when existing in combination with a second mutation in a separate LQTS locus (e.g., the mild SCN5A A572D mutation coexisting with a more serious KCNQ1 V254M mutation). ${ }^{29}$ Compound heterozygous phenotypes lie midway, in terms of degree of QT prolongation, and frequency and severity of cardiac events, 
between those of RWS (1 functional mutation), and homozygous mutations.

As with JLNS patients, LQT 7 and 8 patients exhibit extracardiac abnormalities. The triad of manifestations stemming from KCNJ2 mutations (LQT7) includes ventricular arrhythmias, periodic paralysis, and skeletal developmental abnormalities (Andersen syndrome; AS). Penetrance is variable. LQT8 is more strikingly multi-systemic, leading to congenital heart disease, syndactyly (depending on the mutation), immune deficiency, intermittent hypoglycemia, developmental delay, and autism (Timothy syndrome; TS).

\section{DISEASE ASSOCIATIONS}

\section{Brugada syndrome, progressive cardiac conduction defect disease (PCCD), and sudden infant death syndrome (SIDS): Other SCN5A-related disorders}

For the disease associations search, we used the results of the gene database searches together with more focused searches. Several SCN5A review articles were also quite useful. ${ }^{30,31}$

Brugada syndrome is characterized by ST-segment elevation in the right precordial leads (V1 to V3) and, in some patients, right bundle-branch block, with propensity for ventricular fibrillation and sudden death, often nocturnally. ${ }^{32}$ The incidence varies between 5 and 66 persons per 10,000 in wellstudied Asian areas, but is thought to be much less in the United States and Europe. In Southeast Asia, where it is endemic (and believed to be a cause of sudden unexplained nocturnal death syndrome (SUNDS)), the disorder shows a male predominance ( $8: 1$ male:female ratio) and an average onset of 40 years (range 2 days to 84 years globally).

Brugada syndrome is distinguished by its electrocardiographic (ECG) profile and for identified mutations, quickly inactivating sodium channel current, contrasting with the persistent noninactivating sodium channel currents of LQT3. SCN5A article reviews list 67 Brugada syndrome mutations overall, of which 49 are distinct to Brugada syndrome alone, the remaining being shared with other SCN5A syndromes. ${ }^{30}$ Four SCN5A mutations - D1114N, ${ }^{14}$ delK1500, ${ }^{31}$ E1784K, ${ }^{14,31}$ and 1795ins $\mathrm{D}^{14}$-exhibit electrophysiologic profiles of both Brugada syndrome and LQTS. A 2002 series by Priori et al. detected SCN5A mutations in only 28/130 (22\%) of Brugada syndrome probands, however, suggesting further genetic heterogeneity. ${ }^{31}$

PCCD, also called Lenegre-Lev's disease, is one of the most common cardiac conduction disturbances, which cause disability in millions of people worldwide, often lead to pacemaker implants, and can be fatal. PCCD is characterized by progressive, age-related slowing of cardiac conduction through the His-Purkinje system, right or left bundle branch block, and prolongation of the PR rather than the QT interval. Investigators have sequenced SCN5A mutations displaying PCCD in combination with LQT3 (delK1500), Brugada syndrome (S1710L, G1406R), atrioventricular block (G298S and D1595N), sick sinus syndrome (G1408R), and even dilated cardiomyopathy (D1275N). Proximal changes in base charac- ter or position can be critical, as a change in one base pair can result either in LQT3 (Y1795C mutation) or Brugada syndrome $(\mathrm{Y} 1795 \mathrm{H}),{ }^{31}$ and a minute positional difference in the same base substitution can lead to either PCCD and Brugada syndrome (G1406R) or PCCD and sick sinus syndrome (G1408R). ${ }^{33}$ This subtle mutability illustrates the complexity and relatedness of the various SCN5A-related syndromes.

SIDS, sudden unexpected infant death below 1 year of age, is a multifactorial phenomenon with numerous putative causes, including LQTS. The incidence of SIDS is 1.6 per 1,000 live births in the United States, and 0.7 per 1,000 in Italy. Several studies have documented significant differences in mortality rate between newborns with and without a relatively prolonged QT interval. In one large Italian study $(\mathrm{N}>33,000)$, $50 \%$ of the infants who died of SIDS had a QTc $>440$ milliseconds, which increased the risk of SIDS by a factor of 41.3 (95\% C.I.: 17.3-98.4). ${ }^{34}$

LQTS-specific mutations in the SCN5A gene-S941N, A997S, A1330P, M1766L, R1826H-have been detected in aborted near-death and postmortem SIDS cases by several investigative teams, with one group (Ackerman et al.) estimating an LQTS prevalence of 2\% in SIDS cases. ${ }^{35}$ Evidence also exists that phenotypically severe Brugada syndrome mutations such as L567Q may be involved in SIDS. However, the LQTS association is further strengthened by the observation of SIDS cases bearing KCNQ1 (P117L) and HERG (K101E) mutations. Despite these findings, ECG assessment of sleeping infants has not demonstrated any relation between body position (strongly related to SIDS risk) and QTc interval that might correlate the two factors. ${ }^{36}$

\section{2:1 Atrioventricular (AV) block: Association with SCN5A and HERG}

The 2:1 AV block that can accompany LQTS results from a prolonged electrical recovery time in His-Purkinje and/or ventricular tissues exceeding the sinus cycle length, rather than innately impaired conduction as seen in PCCD. AV blocks in LQTS patients are of great concern because of bradycardiainduced further lengthening of the QT interval to values often in excess of 600 milliseconds, with increased risk of TdP and sudden death. Cases have been reported of SCN5A mutations (G298S, D1595N) possessing inactivation dynamics distinct from both LQTS and Brugada syndrome. However, SCN5A mutations-P1332L, M1677L, V1763M, V1777M (homozygous), and homozygous HERG mutations-L552S, exon 4 bp558-600 duplication, have also been documented in instances of 2:1 AV block in families with LQTS.

\section{Familial atrial fibrillation and short QT syndrome: Associations with KCNQ1, HERG, and KCNJ2}

Atrial fibrillation has a mean prevalence of $0.89 \%$ in the U.S., increasing to $5.9 \%$ over age 65 . It accounts for one-third of strokes in patients over age 65. An initial study by Brugada et al. of a family in Spain and two other smaller families suggested possible linkage to chromosome 10q22-q24. ${ }^{37}$ A 2003 study of a four-generation family with familial atrial fibrillation in Shandong Province, China implicated the KCNQ1 S140G missense mutation, with 
QTc ranging from 450 to 530 milliseconds. ${ }^{38}$ However, atrial fibrillation as well as inducible ventricular fibrillation have been observed in several European families with QTc $\leq 260$ milliseconds, characteristics of the short QT syndrome. ${ }^{39}$ Reports are emerging of further possible associations with the LQT1, 2, and 7 genotypes.

\section{Asthma: A pulmonary association}

A retrospective analysis of 713 LQTS families enrolled in the International LQTS Registry has also disclosed an association between LQTS and asthma, identified in 220 (5.2\%) of 4,310 family members. ${ }^{40}$ The study uncovered an almost twofold higher prevalence of asthma among LQTS-affected patients than their borderline QT interval or unaffected family members (7\% vs. $4 \% ; P<0.001)$. The investigators controlled for the effects of beta-blocker medications.

\section{Clinical disorders with secondary (acquired) LQTS}

QT prolongation associated with heart failure is a common, acquired form of the syndrome. Large-scale epidemiologic studies have demonstrated an association between QTc prolongation and cardiac prognosis in patients with heart disease, though many studies lack appropriate adjustment for prior conditions. QT interval prolongation has also been described in cirrhosis (with and without alcohol), and in acute conditions such as myocardial ischemia, hypothermia, hypothyroidism, pheochromocytoma, and subarachnoid hemorrhage.

\section{INTERACTIONS WITH RISK FACTORS}

\section{Gene-gene interactions}

Investigators have noted variable QTc and ST-T-wave patterns in individuals having the same LQT1-3 genotype and among family members with the same mutation, suggesting involvement of modifier genes or other interacting factors. ${ }^{6}$ Empirical evidence for gene-gene interactions comes from observations of different protein subunits and of multiple allelic variants operating together. In vitro studies co-expressing mutant and wild-type potassium ion channel proteins, some involving mutations or polymorphisms in the beta regulatory subunit, have yielded reductions in potassium channel current densities between 28 and $97 \% .^{41}$

Some interactions may be relatively common. For example, the SCN5A Q1077del ubiquitous splice variant (estimated population frequency: 65\%) may coexist with the SCN5A H558R polymorphism present in heterozygous form in approximately $30 \%$ of individuals in the general population, and in homozygous form in approximately $5 \%$ of individuals. $\mathrm{I}_{\mathrm{Na}}$ current densities, together with a more positive voltage dependence of inactivation, may be reduced $17.5 \%$ in H558R heterozygotes and $35 \%$ in homozygotes also possessing the splice variant. $^{24}$ Several other variants also display either an alteration in current density or shifts in channel activation, inactivation, or recovery in the presence of Q1077 del. In contrast, when H558R coexists in an individual bearing an SCN5A M1766L mutation, the polymorphism rescues the latter muta- tion from causing a trafficking defect. ${ }^{42}$ Investigators have proposed a "double hit" hypothesis for the compound effect of different LQTS gene alterations.

\section{Triggers for Torsade de Pointes in congenital LQTS}

A 2001 survey by Schwartz et al. of 670 patients in the International LQTS Registry revealed genotype-specific patterns in the type of trigger responsible for LQTS cardiac events. ${ }^{5}$ Sixtytwo percent of events in LQT1 patients occurred during exercise, especially swimming, and $43 \%$ of events in LQT2 patients were connected with episodes of emotional stress (fear, anger). In addition, sudden intense auditory stimuli, e.g., elicited by alarm clocks or phones, often accompanied LQT2 cardiac events. LQT3-associated arrhythmic attacks occurred predominantly during rest or sleep (39\%). Catecholaminergic polymorphic ventricular tachycardia (CPVT), a non-LQTS arrhythmic disorder, must be ruled out in some cases of exertional cardiac events.

\section{Sex and age-sex modulation of phenotype}

Sex is an important intrinsic factor in disease etiology and cardiac risk stratification for LQTS patients and their families. Groundbreaking work by Hashiba in Japan revealed a preponderance of females among RWS patients; greater QT prolongation and more severe outcomes in females; and earlier onset of syncope in males. ${ }^{43}$ Family studies have shown that QTc tends to shorten in males during adolescence, with no corresponding changes in female QTc intervals during this period. Research pointing to a hormonal influence has demonstrated gender differences through time in risk and severity of cardiac events according to genotype. ${ }^{26}$ Androgenic protective effects, as well as relative progesterone and estrogen levels in females may be involved. Sex modulates response to QT-prolonging drugs and has been postulated as a modifier of gene expression (e.g., QTc in HERG K897T polymorphisms). ${ }^{44}$

\section{Drug-induced LQTS}

Acquired LQTS is a potentially life-threatening problem that is international in scope. The incidence of TdP with antiarrhythmic use varies from $0 \%$ to $8 \%$ depending on the particular drug and, in some cases, dose. Much less commonly, certain types of noncardiac medications-antipsychotics (e.g., thioridazine), methadone, antimicrobials (e.g., erythromycin), the gastrointestinal stimulant cisapride, and antihistamines (e.g., terfenadine)-may induce TdP.

QT-prolonging drugs characteristically directly block $\mathrm{I}_{\mathrm{Kr}}$ potassium channels, creating an "LQT2" phenotypic equivalent. In exceptional instances, the drugs may interfere with HERG protein trafficking. One study estimated the odds ratio for cardiac events at 1.93 (95\% CI: 1.89-1.98) for each unit increase in HERG blocking activity. ${ }^{45}$ Drug effect on potassium channels may also be potentiated by a variety of milieurelated factors, such as ancillary drug use, ${ }^{1}$ hypocalcemia, hypokalemia and hypomagnesemia related to diet, medical conditions, ${ }^{19}$ diuretic use, and recent cardioversion of atrial fibrillation causing sudden heart rate slowing. 
Research teams have uncovered mutations in several LQTS genes that could predispose individuals to drug-induced TdP: KCNQ1-Y315C, R555C, R583C23; HERG-P347S, R784W23; SCN5A-L1825P, S1102Y25; KCNE1-V109I'13; and KCNE2A116V, M54T, I57T. In a study of 92 patients exhibiting marked drug-induced QT prolongation, Yang et al. were able to identify allelic variants in $10-15 \%$ of affected individuals. ${ }^{23} \mathrm{~A}$ concern is that phenotypically normal individuals harboring "forme fruste" (clinically inapparent, low penetrance) mutations may escape detection and be exposed to potassium channel blocking drugs at some point, placing them at risk for TdP. ${ }^{27}$ These concerns are of great importance for drug development and its regulation by government agencies and the pharmaceutical industry.

\section{CLINICAL EVALUATION AND LABORATORY TESTING}

\section{Phenotypic ascertainment: Clinical and baseline ECG diagnosis}

A clinical scoring system (where 3 points $=$ an item indicating greatest likelihood of LQTS, such as QTc $\geq 480$ milliseconds; and 0.5 points $=$ an item indicating least likelihood, such as congenital deafness), based on personal and family history, symptomatology, and ECG has been developed. The summed clinical point score (Schwartz score) allows patient classification into low ( $\leq 1$ point), intermediate, and high ( $\geq 4$ points) risk groups. ${ }^{46}$ Supplanting the former less specific 440 milliseconds cutoff value, a QTc exceeding 450 milliseconds in men, or 460 milliseconds in women and children (top 5\% of the normal QTc distribution curve), is considered prolonged. The sensitivity of clinical scoring combined with ECG is around $70 \%$ (Table 3). Computer-generated ECG measurements and interpretation should be accompanied by operator review. ${ }^{6}$

Notched or bifid T-waves may serve as a useful phenotypic marker for certain QTc categories, and appear to be much more frequent in LQT2 than LQT1 and LQT3 patients. ${ }^{6,47}$ Other ST-T-waveform patterns, as described in Table 1, may point to LQT1 or LQT3 variants. ${ }^{6}$ Overt T-wave alternansgrossly visible beat-to-beat variation in T-wave amplitude and/or polarity-is viewed as an important prognostic indicator for TdP and sudden cardiac death in LQTS patients, but appears less frequently $(2.5 \%$ to $7 \%$ of LQTS study participant samples) than microvoltage $\mathrm{T}$-wave alternans (identifiable only by special signal processing- 18 to $45 \%$ of LQTS patient groups undergoing specialized testing). ${ }^{48}$ Another repolarization parameter, QT dispersion-the difference between the maximum and minimum QT values across the 12 standard ECG leads- has led to inconsistent results in distinguishing symptomatic from asymptomatic patient groups and fails to take into account $\mathrm{T}$-wave morphology. ${ }^{49}$

\section{Phenotypic ascertainment: Other diagnostic tests}

Clinicians and investigators use exercise stress testing and 24-hour ambulatory ECG monitoring to increase the ability to identify actual LQTS cases among individuals with QTc prolongation. ${ }^{50}$ Both of these techniques can aid clinicians in distinguishing LQT1 from LQT2 patients. Studies have also shown differential response of LQT1, 2, and 3 patients to epinephrine infusion. Epinephrine challenge is considered a powerful provocative test in the unmasking of low-penetrance KCNQ1 mutation carriers. ${ }^{51} \mathrm{~A}$ number of nonpharmacologic positional and other maneuvers affecting autonomic tone have also been studied, to a less rigorous degree, as possible inducers of the LQTS phenotype.

\section{Genetic testing}

Genetic testing has so far largely been relegated to the research setting, making the phenotypic and nonmolecular genotypic tests cited above the mainstay of clinical risk assessment. The recent commercial marketing of short turn-around time ( $\sim 6$ weeks) long QT syndrome genetic diagnostic testing, e.g., Genaissance Pharmaceutical's FAMILION Test, and increasing availability of testing through university-affiliated laboratories in several countries, could establish genetic testing as a clinical tool. However, before widespread use, the clinical (as opposed to analytic) validity of LQTS genetic testing needs to be more widely established. About $60-75 \%$ of LQTS patients have an identifiable LQTS-causing mutation present in one of the 5 most prevalent cardiac channel genes (KCNQ1, HERG, SCN5A, KCNE1, KCNE2). 14,16,52

Genomic DNA in LQTS mutational analyses is typically derived from peripheral blood lymphocytes, more rarely buccal swabs. LQTS studies generally make DNA-based diagnosis more cost-efficient by employing single-strand conformational polymorphism (SSCP) analysis and denaturing high performance liquid chromatography (DHPLC) as initial screens. In the general genetics literature, SSCP sensitivity for detecting true mutations ranges from 75 to $98 \% .{ }^{53}$ Table 3 provides more specific data relating to LQTS.

As demonstrated by a Mayo clinic trial involving DNA sequencing of samples from a DHPLC-negative subset of LQTS referrals, private and rare mutations may be missed. ${ }^{8}$ The issue of how mainstream LQTS genetic tests will be interpreted-via physician, genetic counselor, or direct consumer marketingneeds to be addressed.

\section{POPULATION TESTING}

\section{Genetic screening of specific populations}

LQTS population screening programs are shaped by existent facilities and whether particular highly prevalent mutations have been identified within a given country. Long QT registries are maintained in Denmark (Statens Serum Institute-mutations-based) and China (ECG-based). The University of Helsinki routinely assesses high prevalence founder mutations. Investigators in countries such as Japan, Belgium, and Germany have conducted systematic screening of clinical and population-based groups for acquired LQTS-related polymorphisms. Genetic screening of particular subgroups showing susceptibility to LQTS could also be a part of future clinical programs. Screening for LQTS genetic variants in particular racial-ethnic groups ${ }^{22,24,25}$ would need to be accompanied by appropriate socio-ethical analysis. 


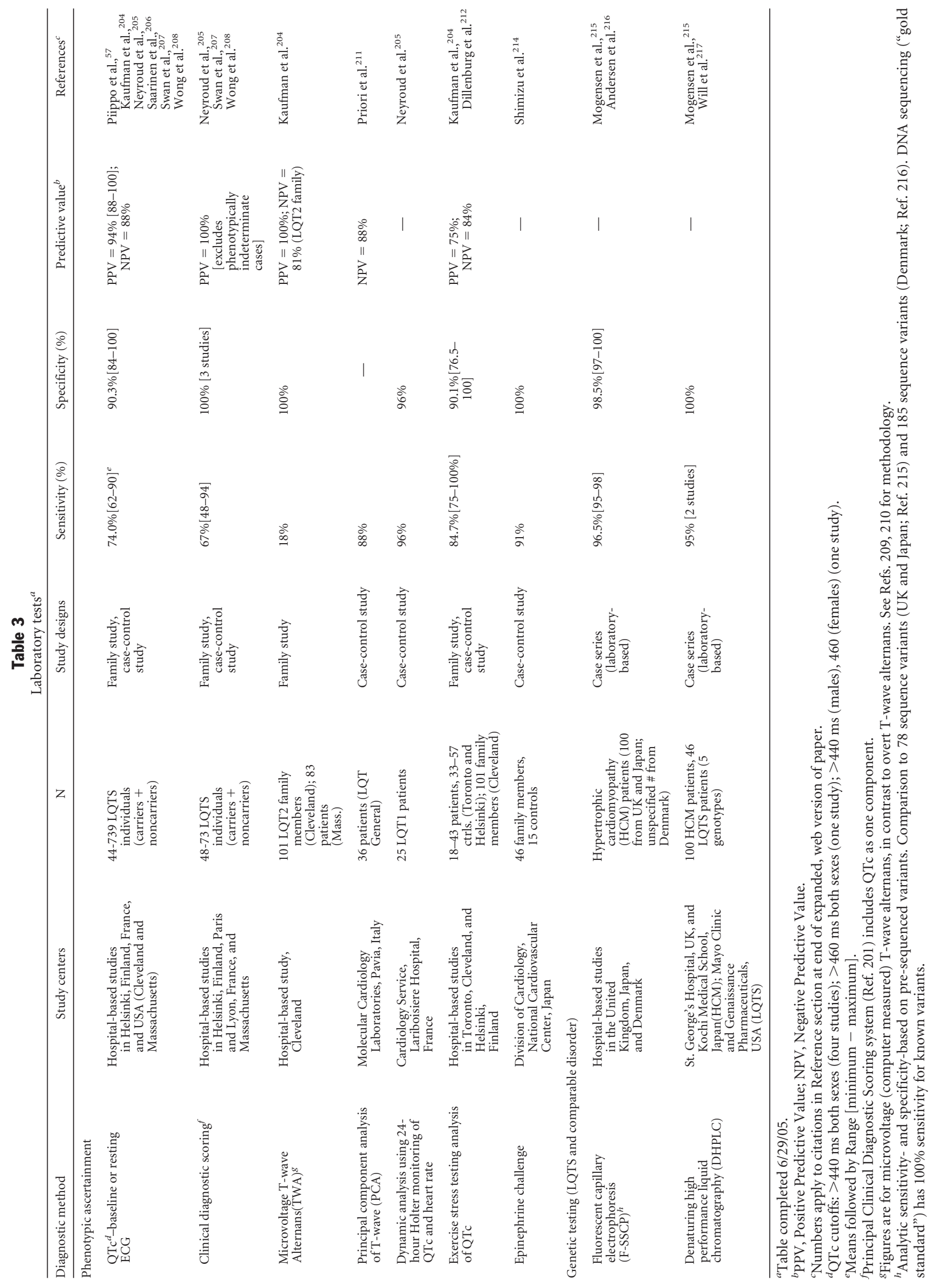




\section{Newborn screening}

Prenatal genetic testing for congenital LQTS has so far occurred only in the investigational setting with limited numbers of cases. Testing for LQTS in newborns typically takes place with referral for ECG abnormalities, such as 2:1 AV block and sinus bradycardia. Preliminary data from a prospective study assessing QTc in 50,000 consecutive neonates has shown that newborn population screening for LQTS is possible, though overall feasibility is uncharted. ${ }^{54}$ While bodies such as the European Society of Cardiology have advocated neonatal ECG inspection as a step toward SIDS prevention, ${ }^{55}$ consensus in this area is not yet established.

\section{Family screening}

Family history can serve as a useful initial screening device to identify potentially at-risk individuals on a population basis. Asymptomatic children and adults with a family history of LQTS, persons who have experienced the unexplained sudden death of a young family member, and relatives of patients with known LQTS should be considered at-risk. Further work-up of at-risk family members may be performed either through mutation testing or ECG. Priori et al. have successfully used a scheme based on QTc, sex, and genotype to divide patients into high, intermediate, and low risk categories. ${ }^{15}$

A perennial obstacle is that gene carriers and non-carriers share a significant region of QTc overlap, particularly in the broad QTc "borderline" band of 420-450/460 milliseconds. Vincent et al. recommend setting the QTc cutoff high-460 milliseconds as applied to general family screening; 490-500 milliseconds when screening large numbers of children-so as to avoid falsely identifying numerous unaffected persons as positive. Syncope as a criterion for initiating ECG analysis in children could reduce the cost per LQTS case detected, but is nonspecific. ${ }^{56}$

DNA analysis is useful for identifying secondary cases within families. In the Netherlands, work-up of family members at five cardiogenetic outpatient clinics results in about 200 families per clinic being seen annually, and has yielded a mean of 3.5 mutation-carrying relatives for each index case. ${ }^{57}$

Van Langen et al. in the Netherlands used decision analysis on data from 31 unrelated LQTS patients to distinguish between three possible genetic screening strategies utilizing: 1) most eligible gene based on reference group prevalences; 2) five major LQTS genotypes; 3) most eligible gene based on individual phenotype. ${ }^{16}$ Strategy 3 proved most cost-effective. Future decision analyses will need to take into account the increasingly observed higher than expected frequencies of compound heterozygous mutations in probands and carrier relatives, which might suggest testing for more than one mutation.

\section{Intervention}

The Task Force on Sudden Cardiac Death of the European Society of Cardiology has developed evidence-based recommendations for the various LQTS interventional modalities involving activity restriction, beta blocker therapy, careful attention to drug use, and invasive approaches. ${ }^{58}$ Sports to be avoided are further detailed in a consensus document developed by the American Heart Association. ${ }^{59}$ Several teams have demonstrated reductions in QT interval length and $\mathrm{T}$-wave abnormalities in LQT2 patients following intravenous and oral potassium administration, though effects may not be long lasting. Class Ic sodium channel blockers may counter the gainof-function abnormality in LQT3, but run the risk of creating a pharmacologic Brugada syndrome phenotype. Innovative techniques such as drug rescue of misfolded ion channel proteins using pharmacological chaperones and ventricular gene therapy are areas of current and future investigation.

\section{OTHER PUBLIC HEALTH APPLICATIONS}

\section{Prevention of sudden cardiac death based on decedent information, provider education}

Clinicians and epidemiologists envision an early warning system for at-risk families. A system based on decedent information could be used to alert surviving family members and relatives having little or no knowledge of arrhythmic death in the family. Progress has been made in this direction with the detection of KCNQ1 and HERG mutations in postmortem samples by several investigative teams. Even in the absence of postmortem tissue for DNA analysis, aggressive clinical and molecular genetic screening of relatives of young SCD victims can identify unsuspected carriers of LQTS gene mutations. In the future, these developments will confront practitioners with new types of ethical-social dilemmas balancing privacy and health concerns. Equally important, surveys of practitioner knowledge concerning QT prolongation and the effects of drug use highlight the role public health and medical care institutions can play in providing professional education that might prevent fatal LQTS-related arrhythmias. ${ }^{60}$

\section{FUTURE DIRECTIONS}

Investigational areas requiring continued and further attention for the prevention and management of LQTS include:

- Identification of novel alleles in LQTS coding and noncoding regions, interacting proteins, and other ion channel genes.

- Better prediction of LQTS disease severity and prognosis in individuals, families, and populations, through continued investigation of the functional effects of pathogenic mutations in vitro, and comparison of affected and control populations in different countries and kindreds.

- Elucidation of the genetic basis of modifying pathways, such as polymorphisms influencing sympathetic tone, and of modifying factors, such as hormonal influences.

\section{CONCLUSION}

LQTS serves as a paradigmatic condition in arrhythmia research and genetic research in general. What is being learned about the multifold pathogenetic mechanisms behind LQTS can be applied to other channelopathies. LQTS research 
bridges phenomena at the molecular genetic, tissue, and systemic levels. ${ }^{3}$ The LQTS family of disorders is highly pleiomorphic yet orderly, serving as a conceptual scaffolding for other complex genetic conditions. Despite the uncertainties and complexities posed by LQTS, action must nonetheless be taken for at-risk patients, family members and groups, since LQTS is potentially fatal. This review of LQTS ends on a positive note, since the exploration of significant disease variants and the determination of socially sensitive policy are moving hand-inhand as commercial screening becomes a real possibility.

Note added in proof: Recently a paper has been published reporting the existence of nonprivate mutations in $\sim 50 \%$ of LQTS probands, a finding that may help to streamline genotypic screening. Napolitano C, Priori SG, Schwartz PJ, et al. Genetic testing in the long QT syndrome: development and validation of an efficient approach to genotyping in clinical practice. JAMA 2005;294:2975-80.

\section{Informational internet sites: Information centers}

Office of Genomics and Disease Prevention (OGDP), Centers for Disease Control and Prevention:

http://www.cdc.gov/genomics

European Long QT Syndrome Information Center:

http://www.qtsyndrome.ch

Drugs That Prolong the QT Interval and/or Induce Torsades de Pointes (hosted by Raymond L. Woosley, MD, PhD):

http://www.torsades.org/druglist.cfm

http://www.ArizonaCERT.org

\section{LQTS registries}

Cardiac Arrhythmias Research and Education (CARE) Foundation Long QT Registry:

http://www.longqt.com/longqtreg.html

Russian National Registry for Patients with Long QT Syndrome:

http://www.medit.ru/Win/present/RLQTS.ppt

\section{Genetic databases}

"Gene Connection for the Heart" LQTS Database, European Society of Cardiology Study Group on Molecular Basis of Arrhythmias, Last updated 5/20/05:

http://pc4.fsm.it:81/cardmoc

"Long QT Syndrome Database," Human Genome Organisation (HUGO), Last updated 5/03:

http://www.ssi.dk/graphics/html/lqtsdb/lqtsdb.htm

Online Mendelian Inheritance in Man (OMIM):

http://www.ncbi.nlm.nih.gov/entrez/

query.fcgi?db=OMIM

\section{Clinical}

Statens Serum Institute, Copenhagen, Denmark:

http://www.ssi.dk/sw13048.asp

Boston University School of Medicine Center for Human

Genetics DNA Diagnostics Laboratory:

http://www.bumc.bu.edu/Dept/

Content.aspx?DepartmentID $=1188$ PageID $=2209$

\section{Commercial}

Genaissance Pharmaceuticals FAMILION Cardiac Ion Channel Mutations Testing:

http://www.familion.com

\section{Consumers and professionals}

Cardiac Arrhythmias Research and Education (CARE) Foundation:

http://www.longqt.com

Sudden Arrhythmia Death Syndromes Foundation:

http://www.sads.org

Long QT Syndrome Support Center:

http://www.long-qt-syndrome.com

\section{ACKNOWLEDGMENTS}

Production of this HuGE Review was supported by the Michigan Center for Genomics and Public Health, under a Centers for Genomics and Public Health grant from the Centers for Disease Control and Prevention and the Association for Schools of Public Health.

\section{References}

1. Liu BA, Juurlink DN. Drugs and the QT interval-caveat doctor. $N$ Engl J Med 2004;351:1053-1056

2. Chiang C, Roden DM. The long QT syndromes: genetic basis and clinical implications. J Am Coll Cardiol 2000;36:1-12.

3. Roden DM, Spooner PM. Inherited long QT syndromes: a paradigm for understanding arrhythmogenesis. J Cardiovasc Electrophysiol 1999;10:1664-1683.

4. Vincent GM. Long QT syndrome. Cardiol Clin 2000;18:309-325.

5. Schwartz PJ, Priori SG, Spazzolini C, Moss AJ, et al. Genotype-phenotype correlation in the long-QT syndrome: gene-specific triggers for life-threatening arrhythmias. Circulation 2001;103:89-95.

6. Zhang L, Timothy KW, Vincent GM, Lehmann MH, et al. Spectrum of ST-T-wave patterns and repolarization parameters in congenital long-QT syndrome: ECG findings identify genotypes. Circulation 2000;102:2849-2855.

7. Shimizu W, Horie M, Ohno S, Takenaka K, et al. Mutation site-specific differences in arrhythmic risk and sensitivity to sympathetic stimulation in the LQT1 form of congenital long QT syndrome: multicenter study in Japan. J Am Coll Cardiol 2004; 44:117-125.

8. Tester DJ, Will ML, Haglund CM, Ackerman MJ. Compendium of cardiac channel mutations in 541 consecutive unrelated patients referred for long QT syndrome genetic testing. Heart Rhythm 2005;2:507-517.

9. Tranebjaerg L, Bathen J, Tyson J, Bitner-Glindzicz M. Jervell and Lange-Nielsen syndrome: a Norwegian perspective. Am J Med Genet 1999;89:137-146.

10. Piippo K, Swan H, Pasternack M, Chapman H, et al. A founder mutation of the potassium channel KCNQ1 in long QT syndrome: implications for estimation of disease prevalence and molecular diagnostics. J Am Coll Cardiol 2001;37:562-568.

11. Fodstad H, Swan H, Laitinen P, Piippo K, et al. Four potassium channel mutations account for $73 \%$ of the genetic spectrum underlying long-QT syndrome (LQTS) and provide evidence for a strong founder effect in Finland. Ann Med Suppl 12004 36:53-63.

12. Yoshida H, Horie M, Otani H, Kawashima T, et al. Bradycardia-induced long QT syndrome caused by a de novo missense mutation in the S2-S3 inner loop of HERG. Am J Med Genet 2001;98:348-352.

13. Schulze-Bahr E, Schwarz M, Hauenschild S, Wedekind H, et al. A novel long-QT 5 gene mutation in the C-terminus (V109I) is associated with a mild phenotype. J Mol Med 2001;79:504-509.

14. Splawski I, Shen J, Timothy KW, Lehmann MH, et al. Spectrum of mutations in long-QT syndrome genes. KVLQT1, HERG, SCN5A, KCNE1, and KCNE2. Circulation 2000;102:1178-1185.

15. Priori SG, Schwartz PJ, Napolitano C, Bliose R, et al. Risk stratification in the long-QT syndrome. N Engl J Med 2003;348:1866-1874.

16. Van Langen IM, Birnie E, Alders M, Jongbloed RJ, et al. The use of genotypephenotype correlations in mutation analysis for the long QT syndrome. J Med Genet 2003;40:141-145 
17. Laitinen P, Fodstad H, Piippo K, Swan H, et al. Survey of the coding region of the HERG gene in long QT syndrome reveals six novel mutations and an amino acid polymorphism with possible phenotypic effects. Hum Mutat 2000;15:580-581.

18. Iwasa H, Itoh T, Nagai R, Nakamura Y, et al. Twenty single nucleotide polymorphisms (SNPs) and their allelic frequencies in four genes that are responsible for familial long QT syndrome in the Japanese population. J Hum Genet 2000;45:182183.

19. Kubota T, Horie M, Takano M, Yoshida H, et al. Evidence for a single nucleotide polymorphism in the KCNQ1 potassium channel that underlies susceptibility to life-threatening arrhythmias. J Cardiovasc Electrophysiol 2001;12:1223-1229.

20. Anson BD, Ackerman MJ, Tester DJ, Will ML, et al. Molecular and functional characterization of common polymorphisms in HERG (KCNH2) potassium channels. Am J Physiol Heart Circ Physiol 2004;286:H2434-H2441.

21. Chen JZ, Xie XD, Wang XX, Tao M, et al. Single nucleotide polymorphisms of the SCN5A gene in Han Chinese and their relation with Brugada syndrome. Chinese Med J 2004;117:652-656.

22. Ackerman MJ, Tester DJ, Jones GS, Will ML, et al. Ethnic differences in cardiac potassium channel variants: implications for genetic susceptibility to sudden cardiac death and genetic testing for congenital long QT syndrome. Mayo Clin Proc 2003; 78:1479-1487.

23. Yang P, Kanki H, Drolet B, Yang T, et al. Allelic variants in long-QT disease genes in patients with drug-associated torsades de pointes. Circulation 2002;105:1943-1948.

24. Ackerman MJ, Splawski I, Makielski JC, Tester DJ, et al. Spectrum and prevalence of cardiac sodium channel variants among black, white, Asian, and Hispanic individuals: implications for arrhythmogenic susceptibility and Brugada/ long QT syndrome genetic testing. Heart Rhythm 2004;1:600-607.

25. Splawski I, Timothy KW, Tateyama M, Clancy CE, et al. Variant of SCN5A sodium channel implicated in risk of cardiac arrhythmia. Science 2002;297:1333-1336.

26. Zareba W, Moss AJ, Locati EH, Lehmann MH, et al. Modulating effects of age and gender on the clinical course of long QT syndrome by genotype. J Am Coll Cardiol 2003;42:103-109.

27. Priori SG, Napolitano C, Schwartz PJ. Low penetrance in the long-QT syndrome: clinical impact. Circulation 1999;99:529-533.

28. Tyson J, Tranebjaerg L, McEntagart M, Larsen LA, et al. Mutational spectrum in the cardioauditory syndrome of Jervell and Lange-Nielsen. Hum Genet 2000;107:499503.

29. Paulussen A, Matthijs G, Gewillig M, Verhasselt P, et al. Mutation analysis in congenital long QT syndrome - a case with missense mutations in KCNQ1 and SCN5A. Genet Test 2003;7:57-61.

30. Moric E, Herbert E, Trusz-Gluza M, Filipecki A, et al. The implications of genetic mutations in the sodium channel gene (SCN5A). Europace 2003;5:325-334.

31. Priori SG, Napolitano C, Gasparini M, Pappone C, et al. Natural history of Brugada syndrome: insights for risk stratification and management. Circulation 2002;105: 1342-1347.

32. Antzelevitch C, Brugada P, Borggrefe M, Brugada J, et al. Brugada syndrome: report of the Second Consensus Conference. Heart Rhythm 2005;2:429-440.

33. Benson DW, Wang DW, Dyment M, Knilans TK, et al. Congenital sick sinus syndrome caused by recessive mutations in the cardiac sodium channel gene (SCN5A). J Clin Invest 2003;112:1019- 1028.

34. Schwartz PJ, Stramba-Badiale M, Segantini A, Austoni P, et al. Prolongation of the QT interval and the sudden infant death syndrome. N Engl J Med 1998;338:1709_ 1714.

35. Ackerman MJ, Siu BL, Sturner WQ, Tester DJ, et al. Postmortem molecular analysis of SCN5A defects in sudden infant death syndrome. JAMA 2001;286:2264-2269.

36. Baker SS, Milazzo AS, Valente AM, Paul IM, et al. Measures of cardiac repolarization and body position in infants. Clin Pediatr (Phila) 2003;42:67-70.

37. Brugada R, Tapscott T, Czernuszewicz GZ, Marian AJ, et al. Identification of a genetic locus for familial atrial fibrillation. N Engl J Med 1997;336:905-911.

38. Chen YH, Xu SJ, Bendahhou S, Wang XL, et al. KCNQ1 gain-of-function mutation in familial atrial fibrillation. Science 2003;299:251-254.
39. Gaita F, Giustetto C, Bianchi F, Wolpert C, et al. Short QT syndrome: a familial cause of sudden death. Ciculation 2003;108:965-970.

40. Rosero SZ, Zareba W, Moss AJ, Robinson JL, et al. Asthma and the risk of cardiac events in the Long QT syndrome. Am J Cardiol 1999;84:1406-1411.

41. Chouabe C, Neyroud N, Richard P, Denjoy I, et al. Novel mutations in KvLQT1 that affect $\mathrm{I}_{\mathrm{ks}}$ activation through interactions with Isk. Cardiovasc Res 2000;45:971-980.

42. Ye B, Valdivia CR, Ackerman MJ, Makielski JC. A common human SCN5A polymorphism modifies expression of an arrhythmia causing mutation. Physiol Genomics 2003; 12:187-193.

43. Hashiba K. Sex differences in phenotypic manifestation and gene transmission in the Romano-Ward syndrome. Ann N Y Acad Sci 1992;644:142-156.

44. Makkar RR, Fromm BS, Steinman RT, Meissner MD, et al. Female gender as a risk factor for torsades de pointes associated with cardiovascular drugs. JAMA 1993;270: 2590-2597.

45. De Bruin ML, Pettersson M, Meyboom RH, Hoes AW, et al. Anti-HERG activity and the risk of drug-induced arrhythmias and sudden death. Euro Heart J 2005;26:590597.

46. Schwartz PJ, Moss AJ, Vincent GM, Crampton RS. Diagnostic criteria for the long QT syndrome. An update. Circulation 1993;88:782-784.

47. Lupoglazoff JM, Denjoy I, Berthet M, Neyroud N, et al. Notched T-waves on Holter recordings enhance detection of patients with LQT2 (HERG) mutations. Circulation 2001;103:1095-1101.

48. Kaufman ES, Priori SG, Napolitano C, Schwartz PJ, et al. Electrocardiographic prediction of abnormal genotype in congenital long QT syndrome: experience in 101 related family members. J Cardiovasc Electrophysiol 2001;12:455-461.

49. Malik M. QT dispersion: time for an obituary? Eur Heart J 2000;21:955-957.

50. Swan H, Viitasalo M, Piippo K, Laitinen P, et al. Sinus node function and ventricular repolarization during exercise stress test in long QT syndrome patients with $\mathrm{Kv}$ LQT1 and HERG potassium channel defects. J Am Coll Cardiol 1999;34:823-829.

51. Shimizu W, Noda T, Takaki H, Kurita T, et al. Epinephrine unmasks latent mutation carriers with LQT1 form of congenital long-QT syndrome. J Am Coll Cardiol 2003; 41:633-642.

52. Tester DJ, Will ML, Haglund CM, Ackerman MJ. Effect of clinical phenotype on yield of long QT syndrome genetic testing. Heart Rhythm 2005;2(5 Suppl 1):S46.

53. Scoggan KA, Bulman DE. Single-strand conformational polymorphism analysis (SSCP) and sequencing for ion channel gene mutations. Methods Mol Biol 2003;217: 143-151.

54. Stramba-Badiale M, Goulene K, Bosi G, Bini R, et al. The role of neonatal electrocardiography in the early identification of genetic arrhythmogenic disorders and congenital cardiovascular diseases: prospective data from 21,000 infants. (Abstract) Circulation Suppl 3 2004;110: III-407.

55. Schwartz PJ, Garson A, Paul T, Stramba-Badiele M, et al. Guidelines for the interpretation of the neonatal electrocardiogram. Eur Heart J 2002;23:1329-1344.

56. Allan WC, Timothy K, Vincent GM, Palomaki GE, et al. Long QT syndrome in children: the value of the rate corrected QT interval in children who present with fainting. J Med Screen 2001;8:178-185.

57. Van Langen IM, Hofman N, Tan HL, Wilde AA. Family and population strategies for screening and counseling of inherited cardiac arrhythmias. Ann Med 2004;36 Suppl 1:116-124.

58. Priori SG, Aliot E, Blomstrom-Lundqvist Bossaert L, Breithardt G, et al. Task Force on Sudden Cardiac Death of the European Society of Cardiology. Eur Heart J2001;22:13741450.

59. Maron BJ, Chaitman BR, Ackerman MJ, de Luna AB, et al. Recommendations for physical activity and recreational sports participation for young patients with genetic cardiovascular diseases. Circulation 2004;109:2807-2816.

60. Viskin S, Rosovski U, Sands AJ, Chen E, et al. Inaccurate electrocardiographic interpretation of long QT: the majority of physicians cannot recognize a long QT when they see one. Health Rhythm 2005;2:569-574. 